studies. Furthermore, a transmigration assay using SF from all four genotypes and murine endothelioma cells (bEnd.5) as an endothelial barrier was carried out. For more detailed information, SF transmigration was evaluated when endothelial cells were also pre-treated with TNF-alpha, mimicking inflammatory conditions.

Results Lasp-1 expression is upregulated in SF from hTNFtg mice and localises to structures of cell adhesion and invasion. In the scratch assay, a significantly reduced migration rate was detected in Lasp- $1 \%$ SFs after 24 hrs $(-43.7 \%$ versus wt, p < 0.05$)$ and in Lasp $1 \%$ hTNFtg, respectively $(-69.11 \%$ versus $h T N F t g, p<0.05)$. Live cell imaging studies showed a slower migration and striking differences in migration morphology of Lasp $1 \% /$ hTNFtg compared to hTNFtg SF. Furthermore, analyses showed a significant reduction of transmigration of Lasp1 $1 /$ hTNFtg compared to hTNFtg SF that was even enhanced by TNF-alpha stimulation of the endothelial cells.

Interestingly, interbred Lasp $1 \% /$ hTNFtg mice presented milder clinical symptoms and analyses of histopathology revealed less cartilage degradation and less attachment of synovial tissue to the cartilage than hTNFtg mice at an age of 14 weeks.

Conclusions Our data provide that the migratory capacity of SF is regulated by Lasp-1 and influences the severity of arthritis in hTNFtg mice. SF - when activated - migrate through the formation of invasive and adhesive membrane structures such as invadopodia, where Lasp-1 is prominently localised. Thus, targeting Lasp-1 may be a promising strategy to reduce the invasive and migratory behaviour of synovial fibroblasts in RA.

\section{A8.16 THE ROLE OF ADIPOCYTOKINES IN OSTEOARTHRITIS OSTEOPHYTE FORMATION}

doi:10.1136/annrheumdis-2013-203222.16

${ }^{1} \mathrm{~S}$ Junker, ${ }^{\mathrm{G}} \mathrm{K}$ Krumbholz, ${ }^{1} \mathrm{KW}$ Frommer, ${ }^{2} \mathrm{~S}$ Rehart, ${ }^{1} \mathrm{U}$ Lange, ${ }^{3} \mathrm{~J}$ Steinmeyer, ${ }^{4} \mathrm{M}$ Rickert, ${ }^{5} \mathrm{G}$ Schett, ' $\mathrm{U}$ Müller-Ladner, 'E Neumann. 'Dept Internal Medicine and Rheumatology, Justus-Liebig-University Giessen, Kerckhoff-Klinik Bad Nauheim, Germany; ${ }^{2} D e p t$ Orthopedics and Trauma Surgery, Markus-Hospital, Frankfurt, Germany; ${ }^{3}$ Dept Experimental Orthopedics, University Hospital Giessen and Marburg, Giessen, Germany; ${ }^{4}$ Dept Orthopedics and Orthopedic Surgery, University Hospital Giessen and Marburg, Giessen, Germany; ${ }^{5}$ Department of Internal Medicine 3, Rheumatology and Immunology, Friedrich-Alexander-University of Erlangen-Nürnberg, Erlangen, Germany

Background and Objectives Although obesity is an established risk factor in osteoarthritis $(\mathrm{OA})$, there is limited information about the role of adipose tissue derived factors in bone formation. Adipocytokines such as adiponectin, resistin, and visfatin, are known to be associated with the pathogenesis of rheumatoid arthritis (RA) and OA. Adipocytokines are locally produced in RA and OA joints by osteoblasts, osteoclasts, and chondrocytes. In contrast to their joint-destructive effects in RA, the role of adipocytokines in OA bone remodelling and osteophyte formation is unclear. Therefore, the adipocytokine expression during osteophyte development and in cells of bone formation was analysed as well as their effect on these cells.

Methods Osteophytes, cartilage, and osteoblasts were obtained from $O A$ patients during joint replacement surgery. Serial sections of bone tissue were stained (Masson trichrome, TRAP) and scored from grade one (no ossification, mainly connective tissue and cartilage) to five (ossified mineralised osteophytes, $<10 \%$ connective tissue, ossified remodelling zones). Immunohistochemistry against alkaline phosphatase, collagen-type II, adiponectin, resistin, and visfatin was performed. OA osteoblasts were stimulated with adiponectin or resistin and immunoassays for IL-6, IL-8, and MCP-1 were performed.

Results All adipocytokines were detectable in cultured osteoblasts and all osteophyte grades. In non ossified osteophytes (grade 1), especially adiponectin and to a lower extent resistin and visfatin were detectable in connective tissue fibroblasts. In ossified osteophytes (grade 2-5), resistin and visfatin and to a lower extend adiponectin were expressed by osteoblasts and resistin and visfatin by osteoclasts. In all osteophyte grades adiponectin was detectable in blood vessels and visfatin was found in about $50 \%$ of the chondrocytes.

Osteoblast stimulation with adiponectin increased the release of the inflammatory mediators IL-6 (2.6-fold), IL-8 (4.9-fold), and MCP-1 (2.1-fold). In contrast, resistin led to a non-significant decrease of these factors. The osteoblast populations showed individual differences in the baseline expression of the analysed factors and in their responsiveness to adipocytokines.

Conclusions The adiponectin and visfatin expression in osteophyte connective tissue and cartilage suggests their involvement in early osteophyte development. Resistin and visfatin in osteoblasts and osteoclasts in ossified osteophytes indicates a role in osteophyte formation at later stages. The stimulation of osteoblasts with adiponectin induces the release of inflammatory mediators. Therefore, the analysed adipocytokines most likely are involved in osteophyte formation at different stages and correspondingly affect cells of cartilage and bone formation to a different extent.

Funded by the ANCYLOSS project of the German Ministry of Research and Education (BMBF).

\section{A8.17 THE ROLE OF CXCR2 SIGNALLING IN ARTICULAR CARTILAGE HOMEOSTASIS}

doi:10.1136/annrheumdis-2013-203222.17

1,2 J Sherwood, ' $\mathrm{J}$ Bertrand, ${ }^{2} \mathrm{G}$ Nalesso, ${ }^{2} \mathrm{P}$ Achan, ${ }^{2} \mathrm{C}$ Pitzalis, ${ }^{\top} \mathrm{T}$ Pap, ${ }^{2} \mathrm{~F}$ Dell'Accio. ${ }^{1} / n s t i t u t e$ for Experimental Musculoskeletal Medicine, University Hospital Münster, Münster, Germany; ${ }^{2}$ Centre for Experimental Medicine and Rheumatology, William Harvey Research Institute, Queen Mary University of London, UK

Background and Objectives The production of ELR + CXC chemokines is widely studied in arthritis and is thought to contribute to the inflammatory phenomena that lead to cartilage breakdown. Healthy articular chondrocytes however, also express their own chemokine receptors and ligands, however their function in these cells is puzzling because chondrocytes are encased in a dense extracellular matrix and are not known to migrate in vivo. This study aims to identify the function of this signalling mechanism in articular cartilage.

Materials and Methods Adult human articular chondrocytes were expanded in monolayer culture under standard conditions. CXCR1 and CXCR2 expression was confirmed using semiquantitative reverse transcription polymerase chain reaction (RT-PCR) and Western blotting. Chemokine receptors and ligands were detected in human articular cartilage from healthy and osteoarthritis patients and in mouse articular cartilage using immunohistochemistry. CXCR1/2 signalling was blocked at specific receptor level in human chondrocytes using validated blocking antibodies and siRNA. Chondrocyte phenotypic gene expression was assessed using real time RT-PCR. The content of highly sulphated proteoglycans in chondrocyte micromasses was analysed using Alcian blue staining, guanidine $\mathrm{HCl}$ extraction and spectrophotometric quantification. Surgical destabilisation of the medial meniscus (DMM) was used to induce instability into the left knees of 8 week old CXCR $2 \%$ mice and wild type BALB/C controls ( $\mathrm{N}=10$ per group). Right knees were sham operated as control. 8 weeks following surgery, mice were culled, knee joints were paraffin embedded and sectioned. Representative sections were stained using Safranin orange and osteoarthritis severity was assessed by Chambers scoring.

Results ELR + CXC chemokines and their receptors, CXCR1 and CXCR2 were expressed in normal human articular cartilage. CXCR1 and CXCR2 were expressed in articular cartilage from osteoarthritis 
patients, however their ligands, CXCL8 and CXCL6, were lost from the territorial matrix of chondrocytes. Blockade of CXCR1/2 signalling at receptor level in chondrocytes resulted in a significantly reduced extracellular matrix sulphated glycosaminoglycan content of micromass cultured chondrocytes and a significantly reduced expression of the chondrocyte differentiation markers COL2A1, Aggrecan and SOX9. CXCR2 and CXCL5 were expressed in unchallenged wild type mouse articular cartilage. CXCR2 $\%$ mice subjected to the DMM model and analysed 8 weeks following surgery developed a significantly more severe osteoarthritis phenotype than wild type controls.

Conclusions Our findings indicate that CXCR $1 / 2$ signalling is required for the maintenance of phenotypic stability of articular chondrocytes. We show that mouse CXCR2 signalling is required for articular cartilage homeostasis and is chondroprotective during conditions of challenge in vivo.

\section{A8.18 VISFATIN/PBEF IN BONE REMODELLING OF RHEUMATOID ARTHRITIS}

doi:10.1136/annrheumdis-2013-203222.18

${ }^{1} \mathrm{G}$ Krumbholz, 'S Junker, ${ }^{2} \mathrm{~A}$ Lehr, ${ }^{3} \mathrm{M}$ Rickert, ${ }^{4} \mathrm{G}$ Schett, ${ }^{1} \mathrm{U}$ Lange, ${ }^{2} \mathrm{~S}$ Rehart, 'U Müller-Ladner, 'E Neumann. 'Dept Internal Medicine and Rheumatology, JustusLiebig-University Gießen, Kerckhoff-Klinik Bad Nauheim, Germany; ${ }^{2}$ Dept Orthopedics and Trauma Surgery, Markus-Hospital, Frankfurt, Germany; ${ }^{3}$ Dept Orthopedics and Orthopedic Surgery, University Hospital Gießen and Marburg, Gießen, Germany; ${ }^{4}$ Medical Clinic 3: Rheumatology and Immunology, Erlangen, Germany

Objectives Rheumatoid arthritis (RA) is associated with increased production of the adipocytokine visfatin in synovial fluid and tissue of RA patients. Visfatin promotes the synthesis of pro-inflammatory and matrix-degrading effector molecules in RA synovial fibroblasts. Moreover, an immunohistochemical analysis of RA bone tissue showed a co-localisation of visfatin with key cells of bone remodelling (osteoblasts, osteoclasts) but the role of this adipokine in processes of bone remodelling in RA is unclear. In this study, we focussed on visfatin and its influence on RA osteoblast and osteoclast activity and differentiation as well as on its immunomodulatory properties.

Methods Human osteoblasts and osteoclasts were isolated from bone tissue and blood samples of RA patients and stimulated with visfatin. Visfatin-mediated effects on osteoblasts and osteoclasts were analysed on the transcriptional and translational level using realtime polymerase chain reaction and immunoassays. Additionally, effects of visfatin on matrix-production of osteoblasts as well as differentiation and resorption activity of osteoclasts were examined by Alizarin-Red S-, TRAP- and von Kossa-staining.

Results Stimulation with visfatin induced the secretion of pro-inflammatory cytokines (e.g. IL-6: 5-fold increase; IL-8: up to 100 -fold) in RA osteoblasts. Additionally, quantitative realtime PCR showed several genes being differentially expressed in osteoblasts after stimulation with visfatin (e.g. alkaline phosphatase, OPG, Osterix). In contrast, osteoclasts only weakly respond to visfatin. A regulation on translational level was observed with regard to the production of the cytokines IL- 6 and IL-8, showing a moderate increase.

Conclusions The results of the present study indicate that visfatin influences the activity as well as the differentiation of human osteoblasts in RA by modulating the expression of genes being involved in matrix production and osteoblast phenotype development. These results support the idea of visfatin affecting bone metabolism in RA. Furthermore, the finding of cytokine-induction in osteoblasts and osteoclasts in RA confirm the pro-inflammatory potential of visfatin in RA.

Acknowledgement Funded by the German Research Society (SPP1468, IMMUNOBONE, NE1174/6-1).

\section{Novel therapeutic targets}

\section{A9.1 AVB3 INTEGRIN INHIBITION WITH CILENGITIDE BOTH PREVENTS AND TREATS COLLAGEN INDUCED ARTHRITIS}

doi:10.1136/annrheumdis-2013-203223.1

${ }^{1}$ Despoina Sykoutri, ${ }^{2}$ Nisha Geetha, ${ }^{1}$ Silvia Hayer, ${ }^{1}$ Peter Mandl, ${ }^{1}$ Josef S Smolen, ${ }^{2}$ Gerald Prager, ${ }^{1}$ Kurt Redlich. 'Department of Rheumatology; ${ }^{2}$ Department of Oncology, Medical University of Vienna, Austria

Background Rheumatoid arthritis (RA) is characterised by synovial inflammation and osteoclast (OC) mediated bone erosions. AlphaVbeta3 ( $\alpha v \beta 3)$ integrin is highly expressed in OCs. Av $\beta 3$ blocking antibodies reduce bone resorption and mice lacking $\beta 3$ are osteopetrotic.

Objectives Efficacy testing of the $\alpha v \beta 3$ inhibitor cilengitide, a synthetic Arginine-Glycine-Asparagine amino acid peptide (RGD peptide), on osteoclastogenesis and the collagen induced arthritis (CIA) model for human RA.

Materials and Methods In vitro mouse bone marrow-derived cells (BMCs) were differentiated into tartrate resistant acid phosphatase positive (TRAP+) mononuclear OC precursor cells (pre$\mathrm{OCs}$ ) and TRAP+ multinucleated mature OCs with macrophagecolony stimulating factor (M-CSF) and receptor activator of nuclear factor kappaB ligand (RANKL). Cilengitide, was added in increasing concentrations $(2 \mathrm{nM}$ to $20 \mu \mathrm{M})$ to the culture. These osteoclastogenesis assays were performed on plates coated with RGD containing matrixes osteopontin, fibronectin and fibrinogen but also on Poly-D-lysine to assess $\alpha v \beta 3$ independent adhesion. In vivo CIA was induced in 6-8 week old male DBA/1 mice by immunisation with bovine type II collagen at day 1 , followed by boosting at day 21 . For CIA prevention mice received subcutaneously (s.c.) $1.5 \mathrm{mg} / \mathrm{kg}$ cilengitide $(n=15)$ or placebo $(n=15), 5$ days per week, 1 day prior to CIA induction until day 53. For CIA treatment, mice with arthritis were randomised and received $1.5 \mathrm{mg} / \mathrm{kg}$ (low dose, $\mathrm{n}=19$ ) or $75 \mathrm{mg} / \mathrm{kg}$ (high dose, $\mathrm{n}=7$ ) cilengitide or placebo $(\mathrm{n}=21)$, until day 59. Preventive and treatment effects were evaluated by assessing paw thickness and grip strength.

Results In vitro increasing concentrations of cilengitide (IC50: $250 \mathrm{nM}$ ) dose-dependently reduced pre-OCs on all coatings, indicating early inhibition at the pre-OC proliferation stage. OCs were reduced above $200 \mathrm{nM}$, followed by complete disappearance above $2 \mu \mathrm{M}$. At $200 \mathrm{nM}$ an intriguing morphological difference with reduction in OC size suggested that cilengitide may disrupt spreading and fusion capacity at the early pre-OC stage. In vivo CIA prevention with cilengitide effectively reduced incidence $(92.8 \%$ versus $40 \%$ ) and severity of arthritis as evidenced by reduction of clinical disease activity scores. Low and high dose cilengitide effectively inhibited progression of established arthritis.

Conclusions Osteoclastogenesis requires intact $\alpha v \beta 3$ integrin function. Systemic $\alpha v \beta 3$ integrin inhibition with cilengitide potently prevents and treats experimental CIA. Cilengitide may be a novel therapeutic target in RA.

\section{A9.2 THE PHOSPHOINOSITIDE 3-KINASE PATHWAY REGULATES FIBROBLAST-LIKE SYNOVIOCYTES INVASION}

doi:10.1136/annrheumdis-2013-203223.2

Beatrix Bartok, Gary S Firestein. UC San Diego School of Medicine, La Jolla, CA

Background/Objectives Cartilage destruction mediated by invasive fibroblast-like synoviocytes (FLS) plays a central role in pathogenesis of RA. Increased cell migration and degradation of extracellular matrix are fundamental to these processes. The Class I phosphoinositide 3-kinases (PI3K) control cell survival, proliferation and migration, which might be involved with cartilage damage in 